\section{Efeito da sobreposição de um modelo de obesidade neuroendócrina experimental e hipertensão arterial sobre a pressão arterial, peso corporal e parâmetros metabólicos e renais de ratos}

Effects of the overlapping between an experimental model of neuroendocrine obesity with arterial hypertension under blood pressure, body weight and metabolic and renal parameters in rats

\begin{abstract}
Autores
Lara Beatriz Delfino

Ferreira $^{1}$

Mario Luis Ribeiro

Cesaretti ${ }^{2}$

Aline Francisco

Voltera ${ }^{1}$

Milton Ginoza ${ }^{1}$

Osvaldo Kohlmann

Junior ${ }^{1}$

${ }^{1}$ Laboratório de Hipertensão Arterial da Universidade Federal de São

Paulo - UNIFESP.

${ }^{2}$ Laboratório de

Hipertensão Arterial da

UNIFESP;

Departamento de

Ciências Fisiológicas da

Pontifícia Universidade

Católica de São Paulo -

PUC/SP.
\end{abstract}

Data de submissão: 06/04/2011 Data de aprovação: 18/07/2011

\section{Correspondência para:} Osvaldo Kohlmann Junior Disciplina de Nefrologia UNIFESP/EPM

Rua Botucatu, 740

São Paulo (SP) - Brasil

CEP: 04023-900

E-mail: kohlmann@hrim.

com.br

\section{Suporte financeiro:}

Fundação de Amparo à

Pesquisa do Estado de São

Paulo e Fundação Oswaldo Ramos.

O referido estudo foi realizado na UNIFESP.

Os autores declaram a inexistência de conflitos de interesses.

\section{Resumo}

Introdução: A elevação do índice de massa corporal e a presença de síndrome metabólica se associam com diminuição da função renal e o aparecimento de doença renal terminal. Objetivo: Avaliar o efeito da sobreposição de um modelo de obesidade experimental e hipertensão arterial sobre a pressão arterial, peso corporal e parâmetros metabólicos e renais de ratos. Métodos: Foram estudados ratos machos das cepas Wistar e espontaneamente hipertensos (SHR). Os grupos MSG receberam glutamato monossódico no período neonatal (WST + MSG e SHR + MSG). Os animais controles receberam salina no período neonatal (WST e SHR). Após completarem três meses de vida, por 12 semanas foram pesados e tiveram a pressão arterial de cauda aferida semanalmente. A determinação de microalbuminúria foi realizada nas semanas $0,4,8$ e 12 . Ao final do período de acompanhamento, coletou-se sangue para glicemia de jejum, creatinina e perfil lipídico. Os rins foram retirados, corados e o índice de esclerose glomerular foi calculado. Resultados: A administração de MSG produziu maior ganho percentual de peso corporal, elevação da glicemia de jejum e maior grau de lesão glomerular nos ratos WST - MSG e SHR - MSG quando comparados aos seus controles. Houve maior excreção urinária de albumina nos ratos do Grupo SHR + MSG quando comparados aos SHR. Não houve diferença estatística na pressão arterial de cauda, creatinina e parâmetros do metabolismo lipídico. Conclusões: A associação de obesidade neuroendócrina e a hipertensão arterial promoveram alterações morfológicas e funcionais no glomérulo mais severas do que aquelas observadas nos ratos somente hipertensos.

Palavras-chave: Obesidade. Glutamato de sódio. Ratos endogâmicos SHR. Albuminúria. Glomérulos renais.

\section{Abstract}

Introduction: Increased body mass index and the metabolic syndrome are associated with decreased renal function and the development of end-stage kidney disease. Objective: To evaluate the effect of the overlap between an experimental model of obesity and genetic hypertension on the blood pressure, body weight and metabolic and kidney parameters of rats. Methods: We studied male rats of the Wistar (WST) and spontaneously hypertensive rats (SHR) strains. Monosodium glutamate (MSG) was administered in the neonatal period to both strains, to make up two groups: WST + MSG and SHR + MSG. Animals in the control groups (WST and SHR) received saline. After completing three months of life, a 12-week follow-up period ensued, during which bi-weekly measurements of body weight (BW) and tail-cuff blood pressure (TCBP) were obtained. Microalbuminuria was analyzed at weeks $0,4,8$ and 12 . At the end of the follow-up period, blood was obtained for fasting glucose, plasma creatinine, and lipid profile determinations. The kidneys were removed, stained, and the glomerular sclerosis index was calculated. Results: The administration of MSG produced higher percentage body weight gain, higher fasting blood glucose and a higher degree of glomerular injury in WST-MSG and MSG-SHR rats, compared to their controls. Greater urinary albumin excretion was observed in SHR + MSG rats, when compared to SHR. There was no statistical difference in the TCBP, creatinine, and lipid profile. Conclusions: The association of neuroendocrine obesity and arterial hypertension promoted morphologi$\mathrm{cal}$ and functional changes in the glomerulus. These changes were more severe than those observed in hypertensive-only rats.

Keywords: Obesity. Sodium glutamate. Rats, Inbred SHR. Albuminuria. Kidney glomerulus. 


\section{INTRODUÇÃO}

A obesidade tem se tornado um problema de saúde pública de repercussão global. ${ }^{1}$ Esse quadro vem se agravando devido à alimentação inadequada e ao alto grau calórico associado à diminuição do gasto calórico, o que contribui de modo significativo para a elevação da adiposidade e traz consigo uma série de comorbidades, dentre elas: hipertensão arterial, dislipidemia, diabetes mellitus tipo $2 \mathrm{e}$ doença vascular arteriosclerótica. ${ }^{2}$ A Organização Mundial de Saúde (OMS) estima que, atualmente no mundo, existam aproximadamente 100 milhões de indivíduos obesos. ${ }^{3} \mathrm{O}$ National Health and Nutrition Examination Survey (NHANES II), realizado nos Estados Unidos, revelou que 60 a $75 \%$ dos indivíduos analisados apresentavam sobrepeso ou obesidade. ${ }^{4}$

Recentemente, alguns estudos epidemiológicos confirmaram que existe uma interação entre obesidade, síndrome metabólica e doença renal. ${ }^{5,6} \mathrm{Em}$ várias populações, a elevação do índice de massa corporal e a presença de síndrome metabólica se associam com diminuição da função renal e o aparecimento de doença renal terminal, independentemente da presença de hipertensão arterial e diabetes mellitus. ${ }^{6,7}$

Nos últimos anos, o Laboratório de Hipertensão Arterial da Disciplina de Nefrologia da Universidade Federal de São Paulo (UNIFESP) adquiriu grande experiência em um modelo animal de obesidade neuroendócrina, por meio da administração neonatal de glutamato monossódico associada à hipertensão arterial genética. ${ }^{8,9}$ A administração subcutânea de glutamato monossódico (MSG), um aminoácido neuroexcitatório lesivo ao sistema nervoso central, ${ }^{10}$ resulta na degeneração aguda do núcleo arqueado do hipotálamo que leva, além da obesidade, à disfunção sexual, parada no crescimento, ao déficit comportamental em roedores ${ }^{11}$ e a alterações no controle cardiovascular. ${ }^{12}$ Mais ainda, os animais obesos desse modelo experimental desenvolvem maior deposição de tecido adiposo na região central, possivelmente em virtude de hipercortisolismo. ${ }^{13}$ As lesões geradas pelo MSG produzem obesidade hipotalâmica por meio de diversas alterações metabólicas, como hiperinsulinemia e prejuízo da termogênese. ${ }^{14,15}$

Assim, este trabalho tem como objetivo avaliar o efeito da sobreposição de um modelo de obesidade neuroendócrina experimental e hipertensão arterial sobre a pressão arterial, peso corporal e parâmetros metabólicos e renais de ratos.

\section{Métodos}

Para o presente estudo, foram utilizados ratos machos das cepas Wistar e espontaneamente hipertensos (SHR, spontaneously hypertensive rat), fornecidos pelo Centro de Desenvolvimento de Modelos Experimentais (CEDEME) da UNIFESP. Os animais foram mantidos em condições ideais de biotério na Disciplina de Nefrologia da UNIFESP. O protocolo deste estudo foi revisado e aprovado pelo Comitê de Ética da UNIFESP (protocolo número 0295/04).

Os animais fornecidos pelo CEDEME foram cru-

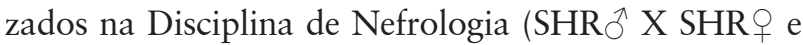
Wistar $\widehat{\supset} \mathrm{X}$ Wistar$\bigcirc$ ) e, no dia seguinte ao nascimento, os filhotes foram separados para que alguns deles recebessem, por via subcutânea, injeções diárias de $2 \mathrm{mg} /$ $\mathrm{kg} / \mathrm{dia}$ (Wistar) e $1 \mathrm{mg} / \mathrm{kg} / \mathrm{dia}$ (SHRs) de MSG até o $11^{\circ}$ dia de vida. Os demais filhotes receberam o mesmo volume de solução fisiológica. Desta forma, os grupos formados foram: Wistar Controle (WST, $\mathrm{n}=10$ ), Wistar + MSG (WST + MSG, $\mathrm{n}=10$ ), SHR Controle $($ SHR, $n=7)$ e SHR + MSG (SHR+MSG, $n=5)$. Os animais permaneceram no biotério da Disciplina de Nefrologia da UNIFESP durante o estudo.

\section{PROTOCOLO EXPERIMENTAL}

Quando os animais completaram três meses de vida, eles passaram a ter, duas vezes por semana, a pressão arterial de cauda mensurada por método oscilométrico e o peso corporal. Os valores médios das duas medidas semanais de pressão arterial de cauda e do peso corporal foram utilizados como representativos daquela semana.

No período basal, na $4^{\mathrm{a}}, 8^{\mathrm{a}}$ e $12^{\mathrm{a}}$ semanas de acompanhamento, os animais foram colocados em gaiolas metabólicas (Nalgene, Rochester, NY) para coleta de urina de 24 horas. Após a medida do volume urinário, realizou-se a determinação de albuminúria de 24 horas pela técnica de imunodifusão radial.

Após o término das 12 semanas de acompanhamento, os animais foram anestesiados com uma solução de Xylasina (10 mg/kg) e Ketamina (75 mg/kg), e um cateter foi introduzido cirurgicamente na artéria femoral. Os animais permaneceram em suas caixas com ração e água à vontade. Nas 12 horas que antecederam o experimento, os animais foram colocados em jejum sólido. Realizou-se a coleta de $1 \mathrm{~mL}$ de sangue por meio do cateter, com o animal tranquilo, para determinação da creatinina plasmática (reação de Jaffé), glicemia de jejum (glicosímetro Accu-Check - Advantage) e de colesterol total e frações e triglicérides (por meio de método enzimático colorimétrico 
automatizado). Por sobrecarga anestésica, os animais foram sacrificados e o rim esquerdo e a gordura periepididimal foram dissecados e pesados em balança analítica. O rim esquerdo também foi fixado em solução de Bouin e depois permaneceu fixado em álcool para posterior determinação do índice de esclerose glomerular.

O índice de esclerose glomerular (IEG) foi realizado após a coloração por hematoxilina-eosina. Um patologista, que desconhecia o grupo de animais, atribuiu graus de lesão glomerular, segundo o Quadro 1.

\section{AnÁlise estatística}

A análise estatística foi realizada por meio da análise de variância de medidas repetidas (one-way repeated measures ANOVA) para comparar as variações temporais da pressão arterial de cauda, do peso corporal e da albuminúria de 24 horas com o período basal. A análise de variância foi utilizada (one-way ANOVA) para comparar as médias de quatro conjuntos de observações, para qual um teste paramétrico é aplicável: glicemia de jejum e peso da gordura visceral. A análise de variância não-paramétrica (ANOVA on RANKS) foi utilizada para a análise estatística do índice de esclerose glomerular. Em todos os testes, fixou-se o nível de significância de $5 \%(\mathrm{p}<0,05)$.

\section{Resultados}

Os resultados estão mostrados na forma de média aritmética, e a dispersão na forma de desvio padrão.

Os dados do peso corporal e da pressão arterial de cauda estão mostrados na Tabela 1.
A análise do peso corporal mostrou que houve um aumento significativo do peso corporal em relação ao período basal nos grupos estudados. Porém, a variação percentual do peso corporal foi significativamente maior nos ratos que receberam MSG no período neonatal $(\mathrm{WST}=15,7 \pm 5,4$

\section{Quadro 1 CLASSIFICAÇÃO DAS LESÕES GLOMERULARES UTILIZADAS PARA CLASSIFICAÇÃO NO ÍNDICE DE ESCLEROSE GLOMERULAR ${ }^{15}$}

Grau 0: Vasos sem alteração ou com redução mínima de seus calibres. Membranas elásticas interna e externa bem definidas, evidenciando relação entre a luz e a parede do vaso superior a 1,5:1,0. Nesse grau, não são visualizados elementos celulares entre o endotélio e a membrana elástica interna.

Grau I: Espessamento hialino da parede vascular em graus variáveis, com manutenção da relação luz/ parede 1,5:1,0. Aumento discreto do número de fibras elásticas na parede do vaso e hipertrofia vascular da túnica média.

Grau II: Redução da luz vascular com redução da relação luz/parede entre 1,5:1,0 e 1,2:1,0. Parede arterial mostrando grande aumento do número de fibras elásticas, inclusive com espessamento, e, por vezes, duplicação da membrana elástica interna. Em alguns casos, tumefação das células endoteliais e presença de gotículas de gordura na camada muscular (degeneração gordurosa).

Grau III: Espessamento concêntrico da parede (aspecto de "casca de cebola"), espessamento mucinoso ou fibroso produzindo acentuada redução do calibre vascular (relação luz/parede $\leq 1,0$ ). Oclusão vascular com ou sem fibrose intramural. Presença de necrose fibrinoide.

TABela 1 Peso CORPORAL, PRESSÃo ARTERIAL dE CAUdA, GLICEMIA E O PERFIL LIPÍDICO

\begin{tabular}{lcccc} 
Variáveis & WST & WST + MSG & SHR & SHR + MSG \\
\hline PCorp Basal (g) & $342,1 \pm 6,6$ & $291,2 \pm 21,9^{*}$ & $257,3 \pm 1,6$ & $218,8 \pm 23,5^{*}$ \\
PCorp 12 Sem (g) & $395,9 \pm 19,2$ & $369,3 \pm 31,3$ & $314,0 \pm 11,6$ & $293,4 \pm 46,9$ \\
$\triangle \%$ Pcorp (\%) & $+15,7 \pm 5,4$ & $+26,9 \pm 7,2^{*}$ & $+23,3 \pm 4,5$ & $+33,5 \pm 6,7^{*}$ \\
PAC basal (mmHg) & $94,4 \pm 9,5$ & $83,9 \pm 7,4$ & $161,9 \pm 6,9^{*}$ & $170,0 \pm 3,5^{*}$ \\
PAC 12 Sem (mmHg) & $101,7 \pm 5,2$ & $93,5 \pm 6,7$ & $192,1 \pm 18,5^{*}$ & $194,4 \pm 11,9^{*}$ \\
$\triangle \%$ PAC (\%) & $+8,38 \pm 2,68$ & $+12,07 \pm 3,5$ & $+18,91 \pm 4,76$ & $+14,32 \pm 2,87$ \\
Glicemia de Jejum (mg/dL) & $65,0 \pm 8,3$ & $82,2 \pm 11,3^{*}$ & $75,4 \pm 2,3$ & $85,5 \pm 2,3^{*}$ \\
Colesterol Total (mg/dL) & $37,5 \pm 16,5$ & $53,2 \pm 17,2$ & $36,4 \pm 6,4$ & $48,0 \pm 13,5$ \\
HDL Colesterol (mg/dL) & $10,7 \pm 3,2$ & $14,4 \pm 3,7$ & $12,4 \pm 2,4$ & $17,2 \pm 1,1$ \\
LDL Colesterol (mg/dL) & $7,9 \pm 2,2$ & $9,4 \pm 2,8$ & $6,7 \pm 1,2$ & $8,4 \pm 3,0$ \\
Triglicerídeos (mg/dL) & $39,3 \pm 11,2$ & $47,0 \pm 14,0$ & $33,3 \pm 6,0$ & $42,0 \pm 15,4$
\end{tabular}

Média \pm desvio padrão do peso corporal (PCorp); variação percentual do peso corporal ( $\triangle \%$ PCorp); pressão arterial de cauda (PAC); variação percentual da pressão arterial de cauda ( $\triangle \% \mathrm{PAC}$ ); glicemia de jejum; colesterol total; HDL-colesterol (mg/dL); LDL-colesterol e triglicerídeos dos grupos WST, WST + MSG, SHR e SHR + MSG; ${ }^{*} p<0,05$ versus WST, ${ }^{*} p<0,05$ versus $S H R,{ }^{8} p<0,05$ versus WST + MSG. 
versus $\mathrm{MSG}=26,9 \pm 7,2 \% *, \mathrm{p}<0,05$ versus WST) $\mathrm{e}$ SHR $=23,3 \pm 4,5$ versus SHR + MSG $=33,5 \pm 6,7 \% *$, $\mathrm{p}<0,05$ versus SHR).

Conforme esperado, a pressão arterial de cauda foi significativamente maior nos ratos espontaneamente hipertensos quando comparados aos Wistar. Não houve diferenças na pressão arterial de cauda entre os grupos tratados ou não com MSG.

A glicemia e o perfil lipídico também estão mostrados na Tabela 1 .

A glicemia de jejum foi significativamente maior nos ratos Wistar e SHR, que receberam MSG no período neonatal, quando comparados aos animais controles, mostrando certo grau de intolerância à glicose nestes animais.

A análise do perfil lipídico não evidenciou quaisquer diferenças estatísticas entre os parâmetros estudados.

A análise dos parâmetros de função renal foi feita pela determinação da creatinina plasmática, da excreção de albumina de 24 horas e da determinação do índice de esclerose glomerular (Figura 1).

Os valores da creatinina plasmática do grupo WST Controle não diferiram daqueles obtidos no grupo SHR (WST $=0,33 \pm 0,14$ versus SHR $=$ $0,38 \pm 0,15 \mathrm{mg} / \mathrm{dL}$, não significante - n.s). Não foi encontrada diferença neste parâmetro nos ratos WST + MSG $(0,28 \pm 0,09$, n.s. versus WST) e, apesar do aumento observado, no Grupo SHR + MSG $(0,53 \pm 0,09$, n.s. versus SHR $)$.

Porém, quando se avaliou a albuminúria na $12^{\mathrm{a}}$ semana de estudo, verificaram-se valores estatisticamente significantes. Quando a análise dos ratos controles foi analisada, verificou-se que os ratos SHR apresentavam valores significantemente maiores de albuminúria quando comparados aos animais controles $(\mathrm{WST}=0,43 \pm 0,08$ versus $\mathrm{SHR}=0,84 \pm 0,11$ $\mathrm{mg} / 24$ horas*, "p $<0,05)$. A administração de MSG aos ratos Wistar produziu elevação da albuminúria, porém sem atingir significância estatística (WST + $\mathrm{DM}=0,60 \pm 0,14 \mathrm{mg} / 24$ horas). No entanto, nos ratos SHR + DM, verificou-se elevação significante da albuminúria (SHR + MSG = 1,14 $\pm 0,23 \mathrm{mg} / 24$ horas, $p<0,05$ versus SHR).

A análise do IEG mostrou que os ratos SHR apresentavam um IEG significativamente maior quando comparado aos ratos do Grupo WST (WST = $0,90 \pm 0,73$ versus $\mathrm{SHR}=2,00 \pm 0,81, \mathrm{p}<0,05)$. A indução de obesidade neuroendócrina aumentou significativamente o IEG nos ratos WST + MSG $(1,70 \pm 0,67, \mathrm{p}<0,05$ versus WST $)$ e SHR + MSG $(2,60 \pm 0,54, \mathrm{p}<0,05$ versus $\mathrm{SHR})$.

\section{Medida da obesidade VISCERAL}

A quantidade de gordura visceral (gordura epididimal) não diferiu significativamente entre os grupos Controles (WST $=0,93 \pm 0,09$ e SHR $=0,98 \pm 0,12$ g/100 g, n.s.). A administração de MSG no período neonatal elevou significativamente o conteúdo de gordura visceral dos grupos WST + MSG $(2,47 \pm 0,17$ $\mathrm{g} / 100 \mathrm{~g}, \mathrm{p}<0,05$ versus WST) e SHR + MSG $(2,12 \pm 0,15 \mathrm{~g} / 100 \mathrm{~g}, \mathrm{p}<0,05$ versus $\mathrm{SHR})$.

Figura 1. Análise de parâmetros da função renal.

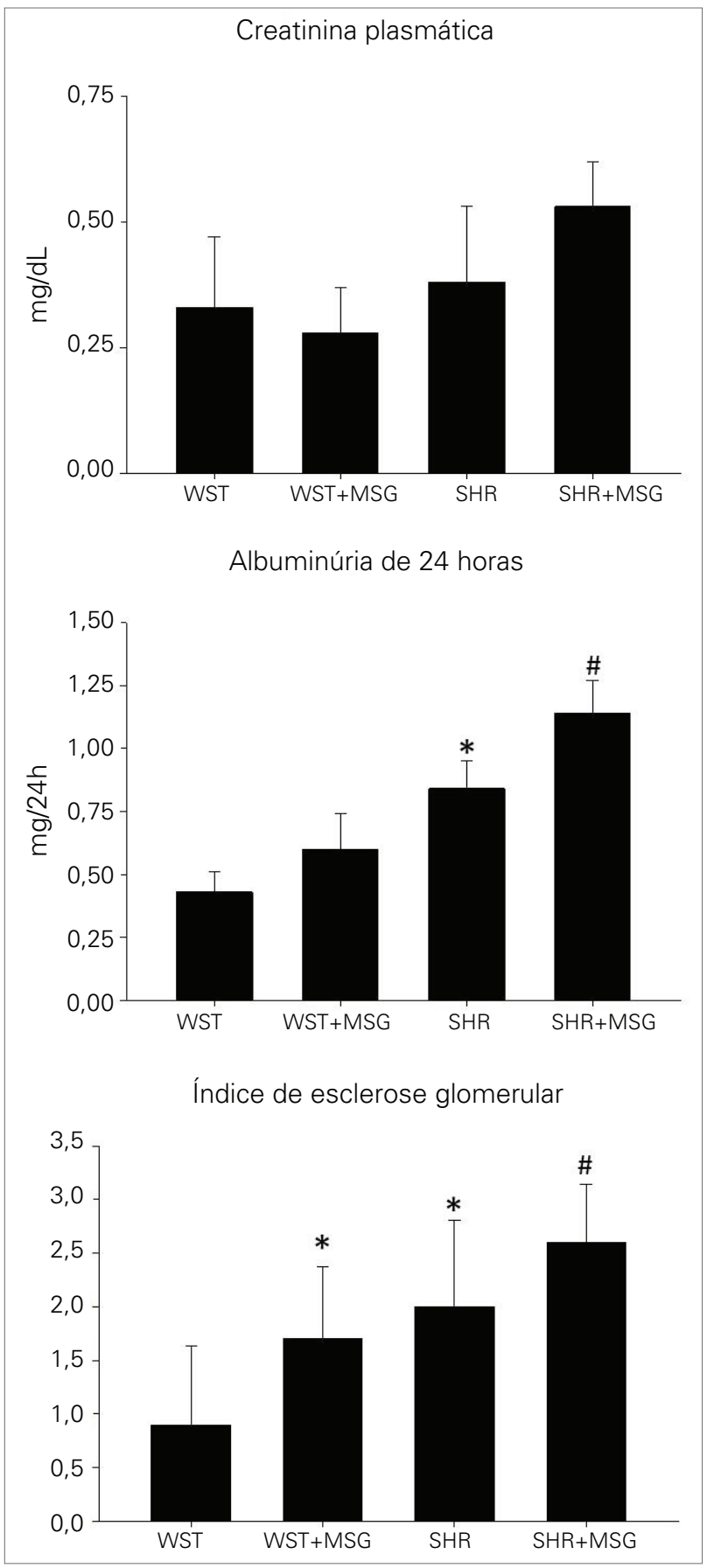

${ }^{*} \mathrm{p}<0,05$ versus WST; ${ }^{\mathrm{p}} \mathrm{p}<0,05$ versus WST + MSG 


\section{Dıscussão}

Este trabalho avaliou a pressão arterial de cauda, a glicemia de jejum, o perfil lipídico e alguns parâmetros da função renal de ratos normotensos e hipertensos, nos quais foi induzida a obesidade neuroendócrina pela administração neonatal de MSG. Apesar de não haver alteração da pressão arterial e do perfil lipídico, os animais desenvolveram alterações estruturais glomerulares, o que refletiu um aumento da albuminúria de 24 horas. A lesão glomerular pode ser atribuída em parte ao aumento da glicemia ou da gordura visceral, que foram verificados neste trabalho.

Neste trabalho, optou-se pelo uso de animais Wistar como controles dos SHR, em detrimento dos ratos Wistar-Kioto. Esta escolha deveu-se ao fato de que ratos Wistar-Kyoto possuem alterações metabólicas e vasculite, portanto, eles poderiam, mesmo em estado de normotensão, ter hipertrofia ventricular. ${ }^{16}$ Ressalta-se que, em outros estudos da literatura, ratos Wistar são utilizados como controles dos ratos SHR. ${ }^{17-19}$

Apesar do menor peso corporal verificado no período basal, os ratos WST + MSG e SHR + MSG tiveram, nas 12 semanas do estudo, um percentual maior de ganho de peso em relação aos animais controles. $\mathrm{O}$ menor peso inicial dos animais MSG deveu-se provavelmente à ação neurotóxica dessa droga, que determina menor secreção de hormônio do crescimento. ${ }^{13}$ Apesar do menor peso corporal, a administração de MSG determinou importante aumento da gordura visceral e da glicemia de jejum sem, no entanto, promover elevação adicional da pressão arterial de cauda. Estudos prévios de nosso laboratório ${ }^{8,9}$, utilizando MSG, demonstraram que em ratos Wistar normotensos houve ganho de peso corporal e diminuição do ganho de peso corporal em SHR.

Em ambos os estudos, não foram verificadas alterações da pressão arterial de cauda. Porém, modelos experimentais de obesidade determinam hiperatividade simpática, secundária ao aumento da gordura visceral e ao hipercortisolismo (devido à administração de MSG), e consequente aumento da pressão arterial de cauda. ${ }^{20}$ Desta maneira, esperava-se neste trabalho elevação da pressão arterial de cauda, o que não ocorreu. No entanto, um estudo realizado por Iwase et al. ${ }^{18}$ mostra dados que corroboram os achados deste trabalho. Esses pesquisadores também administraram MSG no período neonatal a ratos SHR e observaram até redução da pressão arterial de cauda após 14 semanas de vida, que se manteve, porém, em patamares de hipertensão arterial. A justificativa dos autores fundamentou-se no fato de que o MSG leva à interrupção do eixo hipotálamo-hipófise-adrenal, cujo funcionamento deve estar intacto para o desenvolvimento da hipertensão arterial dos ratos SHR. Assim, tomando em conjunto o presente trabalho e os de Iwase et al. ${ }^{18}$, pode-se afirmar que, embora a administração neonatal de MSG ative mecanismos potencialmente pressores, não ocorre sinergismo com aqueles que são ativados na hipertensão arterial experimental espontânea.

O aumento da glicemia de jejum pode sugerir que a intolerância à glicose, apresentada pelos animais dos Grupos WST + MSG e SHR + MSG, já está fundamentada em estudos experimentais. ${ }^{8,13}$ As causas mais prováveis devem-se ao hipercortisolimo que estes animais apresentam, associado à diminuição da capacidade de transporte de glicose por diminuição da atividade dos transportadores GLUT-4 e pelo aumento da gordura visceral em detrimento à gordura marrom. ${ }^{21,22}$

Apesar dos animais deste estudo não apresentarem dislipidemia, um achado importante foi o aumento da gordura visceral secundário à administração de MSG, que caracteriza esse modelo. Associado a esse achado, verificou-se aumento da creatinina plasmática, albuminúria e lesão glomerular nos ratos SHR + MSG e albuminúria e lesão glomerular nos ratos WST + MSG. O aumento da gordura visceral associado à lesão estrutural e funcional glomerular, mesmo em ratos normotensos e hipertensos (em maior intensidade), é o achado mais importante deste trabalho. De fato, em seres humanos, o aumento do índice de massa corporal está associado à diminuição da função renal independentemente da presença de hipertensão arterial e

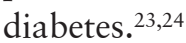

São diversos os fatores que poderiam determinar a lesão glomerular nesse modelo experimental, em que associa-se obesidade neuroendócrina com hipertensão arterial. Trabalhos de Hall et al. ${ }^{21,22}$ propõem que a deposição de gordura visceral poderia determinar compressão da cápsula renal, determinando retenção hidrossalina por ativação do sistema renina angiotensina aldosterona, além do aumento da atividade adrenérgica. Esses dois mecanismos poderiam determinar alteração na hemodinâmica glomerular, promovendo lesão glomerular e o aparecimento de microalbuminúria. O presente trabalho corrobora o estudo de Tamba et al. ${ }^{23}$, os quais verificaram, em pacientes japoneses, que o aumento da gordura visceral per se, como visto nos ratos SHR + MSG, é fator de risco independente para o aparecimento de microalbuminúria. Este autor 
associa a lesão renal de tais pacientes com a diminuição das concentrações plasmáticas de adiponectina.

Outros fatores também podem contribuir potencialmente para o aparecimento da albuminúria. Um dos motivos é a ativação do sistema renina-angiotensina renal. ${ }^{24,25}$ Sabe-se que as concentrações renais de angiotensina II são muito maiores do que os demais órgãos e que, na situação de síndrome metabólica, ocorre ativação desse sistema. ${ }^{25}$ Mesmo que não haja manifestação sistêmica com aumento da pressão arterial, não devem ser desprezadas as ações parácrinas deste peptídeo sobre a hemodinâmica glomerular. Sabe-se que o excesso de AII, por meio de sua ação hemodinâmica, poderia acarretar lesões estruturais nos capilares glomerulares, levando ao aparecimento de albuminúria. ${ }^{26}$

Apesar de não-significante nos ratos MSG deste trabalho, sabe-se que o aumento da adiposidade visceral determina aumento dos níveis circulantes de triglicérides e ácidos graxos, os quais, por sua vez, contribuem diretamente na proliferação das células mesangiais e na produção de citocinas pró-inflamatórias. O processo inflamatório está envolvido com o aparecimento de deposição matriz extracelular com consequente formação de glomeruloesclerose e injúria túbulo intersticial. ${ }^{27,28}$ Essa informação ratifica o achado de que os ratos tornados obesos apresentaram elevação significante do IEG. O estresse oxidativo que decorre do aumento da produção de citocinas inflamatórias, como a interleucina 6, pode determinar o aumento de produtos avançados de glicação (AGE), que por sua vez possui receptores renais, acelerando o processo inflamatório. ${ }^{29}$ É importante ressaltar que o tecido adiposo é uma grande fonte de substâncias próinflamatórias na síndrome metabólica e, dessa forma, poderiam ocorrer lesões inflamatórias no glomérulo. Porém, é importante ressaltar que a leptina pode estimular diretamente a secreção de TGF-beta e levar ao aparecimento de glomeruloesclerose. Porém, neste modelo experimental, essa hipótese deve ser descartada uma vez que a administração de MSG suprime a secreção de leptina. ${ }^{30}$

Dessa forma, pode-se concluir que a associação de obesidade neuroendócrina e a hipertensão arterial promoveram alterações morfológicas e funcionais no glomérulo mais severas do que aquelas observadas nos ratos somente hipertensos. $\mathrm{O}$ aumento da gordura visceral, por determinar aumento de produção de substâncias pró-inflamatórias, bem como fatores hemodinâmicos e hormonais, pode contribuir para acelerar a velocidade de perda da função renal nos ratos hipertensos e tornados obesos.

\section{REFERÊNCIAS}

1. Yanovski SZ, Yanovski JA.Obesity prevalence in the United States--up, down, or sideways? N Engl J Med 2011;364:987-9

2. Allende-Vigo MZ. Pathophysiologic mechanisms linking adipose tissue and cardiometabolic risk. Endocr Pract 2010;16:692-8

3. Stevens J, Cai J, Juhaeri, Thun MJ, Wood JL. Evaluation of WHO and NHANES II standards for overweight using mortality rates. J Am Diet Assoc 2000;100: $825-7$

4. Cohen JD, Cziraky MJ, Cai Q, Wallace A, Wasser T, Crouse JR, et al. 30-year trends in serum lipids among United States adults: results from the National Health and Nutrition Examination Surveys II, III, and 19992006. Am J Cardiol 2010;106:969-75.

5. Masuo K, Lambert GW, Esler MD, Rakugi H, Ogihara T, Schlaich MP. The role of sympathetic nervous activity in renal injury and end-stage renal disease. Hypertens Res 2010;33:521-8.

6. de Boer IH, Katz R, Fried LF, Ix JH, Luchsinger J, Sarnak MJ, et al. Obesity and change in estimated GFR among older adults. Am J Kidney Dis 2009;54: 1043-51.

7. Kiberd B. The chronic kidney disease epidemic: stepping back and looking forward. J Am Soc Nephrol 2006;17:2967-73.

8. Voltera AF, Cesaretti MLR, Ginoza M, Kohlmann Jr. O. Efeito da indução de obesidade neuroendócrina sobre a hemodinâmica sistêmica e a função ventricular esquerda de ratos normotensos. Arq Bras Endocrinol Metab 2008;52:47-54.

9. Ferreira CBND, Cesaretti MLR, Ginoza M, Kohlmann Jr. O. Efeitos da administração de metformina sobre a pressão arterial e o metabolismo glicídico de ratos espontaneamente hipertensos tornados obesos pela injeção neonatal de glutamato monossódico. Arq Bras Endocrinol Metab 2009;53:409-15.

10. Kizer JS, Nemeroff CB, Youngblood WW. Neurotoxic amino acids and structurally related analogs. Pharmacol Rev 1978;29:301-18.

11. Dawson R, Annau Z. A behavioral assessment of arcuate nucleus damage after a single injection of monosodium glutamate. Neurobehav Toxicol Teratol 1983;5:399-406.

12. Dawson R Jr., Wallace DR, Gabriel SM. A pharmacological analysis of food intake regulation in rats treated neonatally with monosodium L-glutamate (MSG). Pharmacol Biochem Behav 1989;32:391-8.

13. Hirata AE, Andrade IS, Vaskevicius P, Dolnikoff MS. Monosodium glutamate (MSG) - obese rats develop glucose intolerance and insulin resistance to peripheral glucose uptake. Braz J Med Biol Res 1997;30:671-4.

14. Pereira LO, Francischi RP, Lancha Jr AH. Obesidade: hábitos nutricionais, sedentarismo e resistência à insulina. Arq Bras Endocrinol Metab 2003;47:111-27.

15. Lemos CC, Mandarim-de-Lacerda CA, Dorigo D, Coimbra TM, Bregman R. Chronic renal failure in male and female rats. J Nephrol 2005;18:368-73.

16. Cesaretti ML, Kohlmann Junior O. Experimental models of insulin resistance and obesity: lessons learned. Arq Bras Endocrinol Metabol 2006;50:190-7. 
17. Iwase M, Yamamoto M, Iino K, Ichikawa K, Shinohara $\mathrm{N}$, Yoshinari $\mathrm{M}$, et al. Obesity induced by neonatal monosodium glutamate treatment in spontaneously hypertensive rats: an animal model of multiple risk factors. Hypertens Res 1998;21:1-6.

18. Iwase M, Ichikawa K, Tashiro K, Iino K, Shinohara $\mathrm{N}$, Ibayashi S, et al. Effects of monosodium glutamateinduced obesity in spontaneously hypertensive rats vs. Wistar Kyoto rats: serum leptin and blood flow to brown adipose tissue. Hypertens Res 2000;23: 503-10.

19. Sarafidis PA. Obesity, insulin resistance and kidney disease risk: insights into the relationship. Curr Opin Nephrol Hypertens 2008;17:450-6.

20. Munkhaugen J, Lydersen S, Widerøe TE, Hallan S. Prehypertension, obesity, and risk of kidney disease: 20-year follow-up of the HUNT I study in Norway. Am J Kidney Dis 2009;54:638-46.

21. do Carmo JM, Tallam LS, Roberts JV, Brandon EL, Biglane J, da Silva AA, et al. Impact of obesity on renal structure and function in the presence and absence of hypertension: evidence from melanocortin-4 receptordeficient mice. Am J Physiol Regul Integr Comp Physiol 2009;297:R803-12.

22. Hall JE, Jones DW, Kuo JJ, da Silva A, Tallam LS, Liu J. Impact of the obesity epidemic on hypertension and renal disease. Curr Hypertens Rep 2003;5:386-92.
23. Tamba S, Nakatsuji H, Kishida K, Noguchi M, Ogawa $\mathrm{T}$, Okauchi $\mathrm{Y}$, et al. Relationship between visceral fat accumulation and urinary albumin-creatinine ratio in middle-aged Japanese men. Atherosclerosis 2010;211:601-5

24. Siragy HM, Carey RM. Role of the intrarenal reninangiotensin-aldosterone system in chronic kidney disease. Am J Nephrol 2010;31:541-50.

25. Vidotti DB, Casarini DE, Cristovam PC, Leite CA, Schor N, Boim MA. High glucose concentration stimulates intracellular renin activity and angiotensin II generation in rat mesangial cells. Am J Physiol Renal Physiol 2004;286:F1039-45.

26. Knight SF, Imig JD. Obesity, insulin resistance, and renal function. Microcirculation 2007;14:349-62.

27. Dey A, Maric C, Kaesemeyer WH, Zaharis CZ, Stewart J, Pollock JS, et al. Rofecoxib decreases renal injury in obese Zucker rats. Clin Sci (Lond) 2004;107:561-70.

28. Henegar JR, Bigler SA, Henegar LK, Tyagi SC, Hall JE. Functional and structural changes in the kidney in the early stages of obesity. J Am Soc Nephrol 2001;12:1211-7.

29. Heidland A, Sebekova K, Schinzel R. Advanced glycation end products and the progressive course of renal disease. Am J Kidney Dis 2001;38(Suppl):S100-6

30. Wolf G, Ziyadeh FN. Leptin and renal fibrosis. Contrib Nephrol 2006;151:175-83. 